\title{
Evolution Balancing of the Small-Sized Wheel Loader Assembly Line
}

\author{
Rongshen Lai, ${ }^{1}$ Liang Hou, ${ }^{1}$ and Yongming $\mathrm{Wu}^{2}$ \\ ${ }^{1}$ Department of Mechanical and Electrical Engineering, Xiamen University, Xiamen, Fujian 361005, China \\ ${ }^{2}$ Key Laboratory of Advanced Manufacturing Technology, Ministry of Education, Guizhou University, Guiyang 550003, China \\ Correspondence should be addressed to Liang Hou; 122774584@qq.com
}

Received 31 December 2014; Accepted 15 February 2015

Academic Editor: Changjun Zheng

Copyright (c) 2015 Rongshen Lai et al. This is an open access article distributed under the Creative Commons Attribution License, which permits unrestricted use, distribution, and reproduction in any medium, provided the original work is properly cited.

\begin{abstract}
Assembly line balancing not only directly determines production efficiency but also influences precision and quality of key assemblies or even the overall performance of final products. Driven by market demand and development of science and technology, product family must evolve constantly, which necessitates frequent adjustment and rebalancing of product family assembly line (PFAL). In order to maintain production efficiency, improve assembly quality and precision, and reduce costs for adjustment, the evolution balancing problem of PFAL for small-sized wheel loader is solved in this paper. Firstly, the evolution balancing model of PFAL is put forward. Then, with minimizing the number of workstations, the in-station and between-station load indexes and adjustment costs, and maximizing relevancy between activities as optimization objectives, meanwhile regarding product platform planning, modularity design and the critical chain technology in concurrent engineering as constraint conditions, the evolution balancing problem of PFAL is optimized using improved genetic algorithm (IGA). Finally, the whole analysis procedure is demonstrated by the small-sized wheel loader PFAL case study and the effectiveness of the proposed method is verified.
\end{abstract}

\section{Introduction}

In most construction machinery manufacturing enterprises, assembly is the last link of production involving many operations and complex processes and has become the core technology. Born in Ford Motor Company, assembly line has greatly improved labor productivity and opened the prelude to modern mass production. However, frequent assembly line adjustment, such as changes of operating personnel, assembly equipment, and material flow, would decrease assembly precision and quality of final products, which further increases difficulty of noise and vibration control [1], faults diagnosis [2-4], bending, and vibration analysis of structural parts, also influences the overall performance of such as suspension systems [5], universal joint in steering handling mechanism [6], and rotated shafts [7].

With customer demand for personalized and diversified product models, such as different requirements for power, safety, comfortableness, and energy conservation, mixed model assembly lines (MMALs) gradually replace the conventional single model assembly lines (SMALs) and become the mainstream assembly mode in the industry of construction machinery. Proposed by Salveson in the 1950s [8], assembly line balancing problem (ALBP) in essence is a combinatorial optimization problem of rationally assigning assembly tasks into workstations and meanwhile minimizing differences of operation time between workstations. ALBP can be divided into two categories, both of which are typical NP-hard problems $[9,10]$ : given the cycle time, minimizing the number of workstations and given the number of workstations, minimizing the cycle time.

Many researchers heretofore have launched in-depth studies on assembly line balancing problem (ALBP), especially on the mixed-model assembly line balancing problem (MMALBP) mainly owing to the capability of MMAL of "producing the needed products in needed quantity at needed time" to effectively improve enterprise competitiveness. Scholl and Becker [11] carried out a comprehensive survey of simple assembly line balancing problem (SALBP) research with a special emphasis on exact and heuristic solution procedures of SALBP. Regarding MMALBP, Merengo et al. [12] 
analyzed some typical problems of manual, mixed-model assembly lines and presented new balancing methodologies pursuing the common goals of minimizing the rate of incomplete jobs in paced lines and in moving lines or the probability of blocking/starvation events (in unpaced lines) and reducing WIP. Haq et al. [13] dealt with mixedmodel assembly line balancing to minimize the number of workstations by incorporating a hybrid genetic algorithm approach which used the solution from the modified ranked positional method as the initial solution to reduce the search space within the global space, thereby reducing search time. Bukchin and Rabinowitch [14] relaxed the restriction that a task that is common to multiple models must be assigned to a single station and developed an optimal solution procedure based on a backtracking branch-and-bound algorithm to minimize the sum of stations and task duplication costs. Özcan and Toklu [15] presented a new mathematical model minimizing the number of mated-stations as the primary objective and the number of stations as a secondary objective for a given cycle time and introduced a simulated annealing algorithm for the mixed-model two-sided assembly line balancing problem. Akpinar et al. [16, 17] proposed a hybrid genetic algorithm sequentially hybridizing Kilbridge and Wester Heuristic, Phase-I of Moodie and Young method and Ranked Positional Weight Technique to solve mixed model assembly line balancing problem of type I with objectives of minimizing the number of workstations, maximizing the workload smoothness between and within workstations. Aiming at stochastic environment of MMALs, Xu and Xiao [18] provided min-max related and a-worst scenario based robust criteria to formulate corresponding optimization models and introduced robust optimization approaches to balance mixed model assembly lines with uncertain task times and daily model mix changes by scenario planning. Yagmahan [19] considered operation time variations together with the number of stations, the line efficiency, and the smooth production to formulate the mixed-model assembly line balancing problem and proposed a more efficient and effective multiobjective ant colony optimization (MOACO) algorithm to solve this problem. Manavizadeh et al. [20] presented a multiobjective genetic algorithm (MOGA) to solve MMALBP in response to different market demands considering cycle time (CT) and the number of stations simultaneously and minimizing capital costs of designing multiple assembly lines and carried out a comparison between six multiobjective evolutionary algorithms (MOEA) to determine the best method for specific situation. Most recently, aiming at the balancing problem of product family assembly line (PFAL), Hou et al. [21] established a balancing model for PFAL and simultaneously proposed an improved dual-population genetic algorithm to solve product family assembly line balancing problem (PFALBP) with minimizing the number of stations, minimizing the load indexes between stations and within each station and maximizing task-related degree are used as optimization objectives optimization objectives.

The related research aforementioned is the basis of assembly line planning and also the key of assembly line balancing. However, most studies are limited to analyzing single-model or mixed-model assembly line balancing problems from static point of view, and research on dynamic evolution process of assembly line is rare, especially the lack of evolution balancing research on modular product family assembly line which is typical in construction machinery manufacturing enterprises. Assembly line evolution would certainly induce adjustment costs, and product family design techniques such as platform, modularity, and commonality would also affect allocation of assembly tasks and assembly line balancing, therefore, aiming at the small-sized wheel loader product family and in order to better guide enterprises to implement product family assembly line planning and innovation we address the dynamic planning of evolution balancing for PFAL based on analysis of product family evolution. Firstly, relation between external influencing factors of product family evolution and assembly line evolution is selectively analyzed. Then, in order to improve assembly efficiency and optimize the assembly line, the precedence graph of assembly tasks is converted into AOE-net by specific rules; the critical tasks and critical path are identified using critical chain technology. Finally, in this paper minimizing the number of workstations, minimizing in-station and between-station load indexes, maximizing task correlation, and minimizing adjustment costs are set as the optimization objectives; the design techniques such as product platform and modularity and critical chain technology are introduced into the evolution balancing model of PFAL, and the evolution balancing problem of PFAL is optimized using improved dual-population genetic algorithm to realize dual objectives of improving assembly efficiency and reducing adjustment costs.

\section{Evolution Balancing Process of PFAL for SWL}

Driven by customer demand, core technology, and market competition, product families evolve constantly [22], which is mainly embodied in the replacement of modules or components and variation in module partition on microscopic level, such as the frame system module in small-sized wheel loader evolving from 915 to $916 \mathrm{~B}$ and to 918 and $918 \mathrm{Te}$; and embodied in the change of product family architecture and configuration difference of product variants on macroscopic level; as shown in Figure 1. There are two situations in the evolution process of product family: gradual improvement and abrupt change. The former includes adjustment of production quantity for different product variants and operations such as addition, removal, modification, and innovation on variant/unique modules in order to accommodate customer requirement differences in function or performance or to implement continuous improvement in product cost or quality, while the latter is mainly due to the emergence of new technology, material, or process which promotes core technology upgrading of product platform or product family.

Product family evolution propels incessant renewal and adjustment of its assembly line. Change of product mix ratio in different periods of time or operation time variation of assembly tasks in new product variants leads the existing 

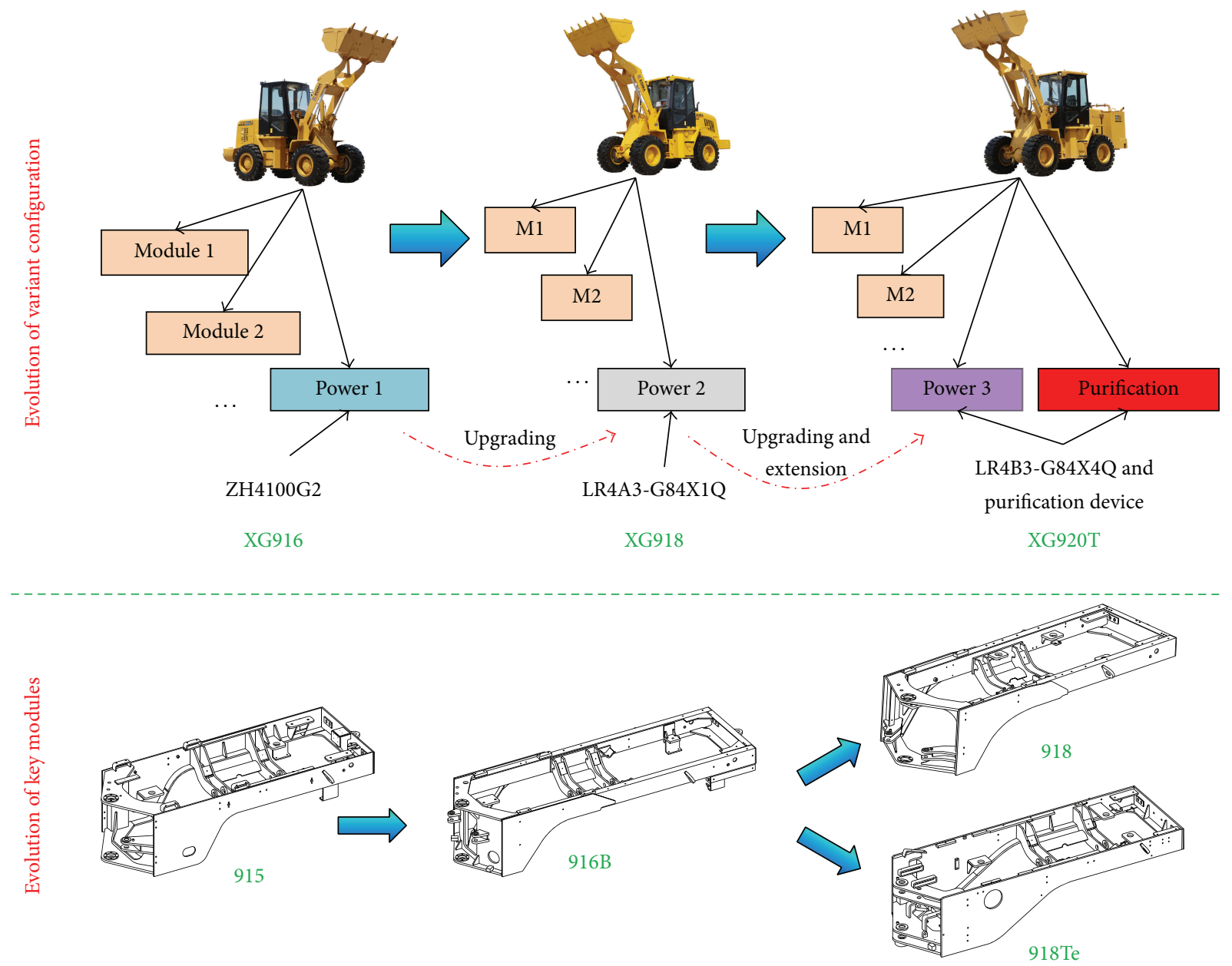

FIGURE 1: Evolution of small-sized wheel loader.

balancing scheme to be no longer valid; in other words, when new products are introduced or operation time of tasks changes, the tasks should be reassigned to maintain load balancing of assembly line, which is defined as evolution balancing problem of assembly line in this paper. Product family assembly line planning must be able to rapidly and efficiently respond to the dynamic evolution of product family such as adding, removing, and upgrading modules and changes of various planning elements such as cost and new technology introduction and fully consider the influence of dynamic quantity change of variant products, task addition or removal, operation time variation, and different solutions of module partition on task assignment and assembly line balancing schemes. In all, product family assembly line must realize balancing in the coevolution process with product family; namely, evolution balancing as shown in Figure 2, share assembly resources between product variants and even generational products to the greatest extent and provide high quality and low cost individualized products in the shortest possible time and reduce the adjustment costs of assembly line $[23,24]$.

\section{Evolution Balancing Model of PFAL for SWL}

In this paper, we focus on the evolution balancing process of modular product family assembly line, where the modular product family refers to a group/series of products oriented to specific market segmentation derived by adding, removing or replacing the variant or unique modules based on product platform. To demonstrate the concepts of product platform and modularity, a simplified example is shown in Figure 3, where the working device is shared by different models and the extinct accessory modules with standard interface can realize specific functions.

Product platform and modularity play an important role in product family development, configuration, production, and so on. Generally, manufacturing enterprises achieve product diversification and customization by means of addition, deletion, and/or modification operations onto variant or unique functional modules and meanwhile try to keep the product platform constant; hence the product platform and key functional modules possess the characteristic of relative 


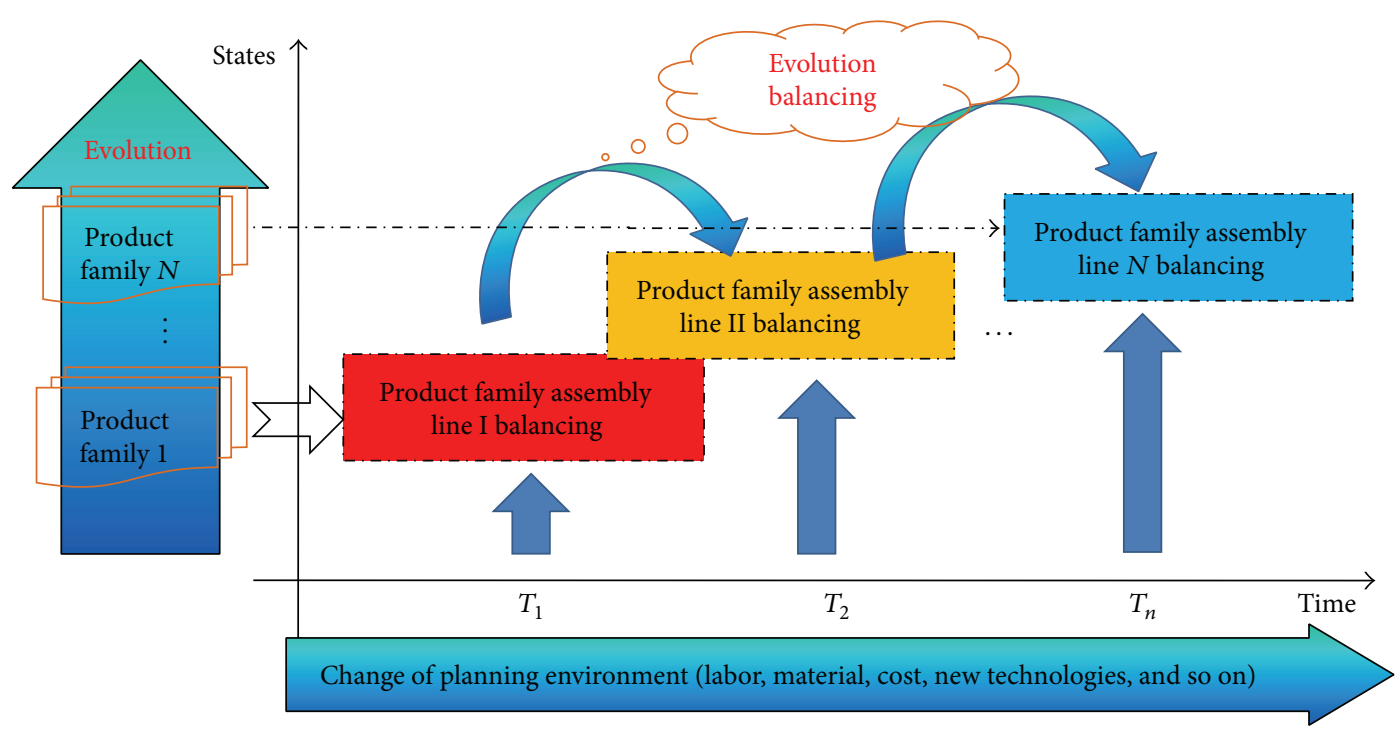

FIGURE 2: Evolution balancing process of PFAL.

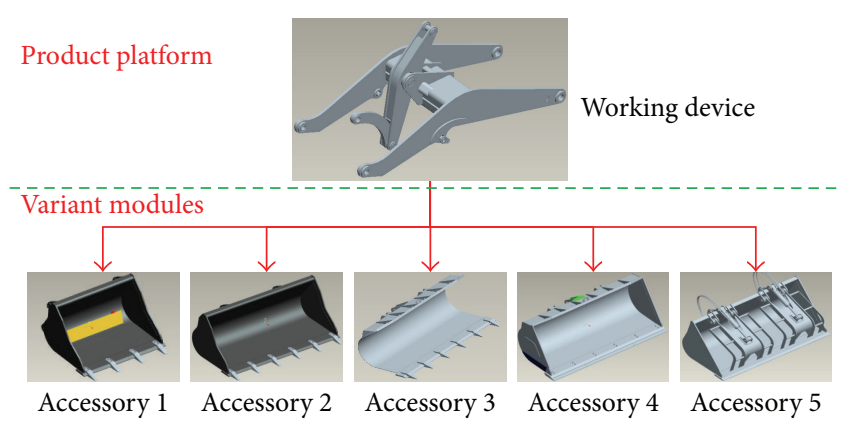

FIGURE 3: Example of product platform and modularity.

higher stability; in other words, the product platform and key functional modules would remain unchanged in the product family evolution process with much higher probability. We take the relative higher stability of product platform and key functional modules into consideration in evolution balancing planning process with the purpose of realizing assembly line balancing as well as minimizing adjustment costs.

In addition, we pay attention to improving assembly efficiency while balancing the assembly line. The critical tasks in assembly process have larger space for improvement and restrict/determine the overall assembly efficiency, and the duration of critical tasks and even the tasks themselves are with relative lower stability compared with product platform and key functional modules; in other words, the duration of critical tasks change frequently and/or greatly and the critical tasks themselves are likely to be replaced by some other tasks. Therefore, we treat critical tasks as flexible planning constraints in the evolution balancing optimization model of PFAL.

3.1. Product Platform/Modularity. Modularity is a prevailing design method based on product functional analysis, in which functional modules are divided according to specific criteria and different products are configured through module selection and combination to meet diversified market demand [25]. Modular design supports mass customization with costs close to that of mass production and finds the best balancing point between product variety, cost, and performance; in essence, it is the process of product structure analysis and restructuring. Principle of module division is to make components in some module with strong correlation, while the components between modules with little correlation. Modular design technique has an important influence on the balancing planning of PFAL, which mainly manifested that correlation between the assembly tasks for a certain module is strong; enterprises usually assemble key functional modules in advance and strictly assign the tasks strongly correlated to some specific functional module into the same station. In this paper, we define task correlation and tend to assign tasks with higher correlation into the same station to reflect the practical assembly situations.

Product platform is defined as "a set of subsystems and interfaces developed to form a common structure from which a stream of derivative products can be efficiently developed and produced" [26], which is an effective development strategy to improve external diversity and reduce internal differentiation by means of adding, deleting, and/or replacing one or several modules based on platform to derive a family of products and meet customers' diversified needs. Once the product platform is determined, it will generally be relatively stable in the gradual evolution process of product family. Therefore, in the evolution balancing planning process of PFAL, the platform-related assembly tasks should be assigned into fixed stations rather than influenced by variations of other tasks, which is conductive to reducing adjustment costs.

Modular product family can be represented by a hierarchical model, as shown in Figure 4, where the functional modules are categorized into common modules, optional modules, and unique modules. Within the same functional 


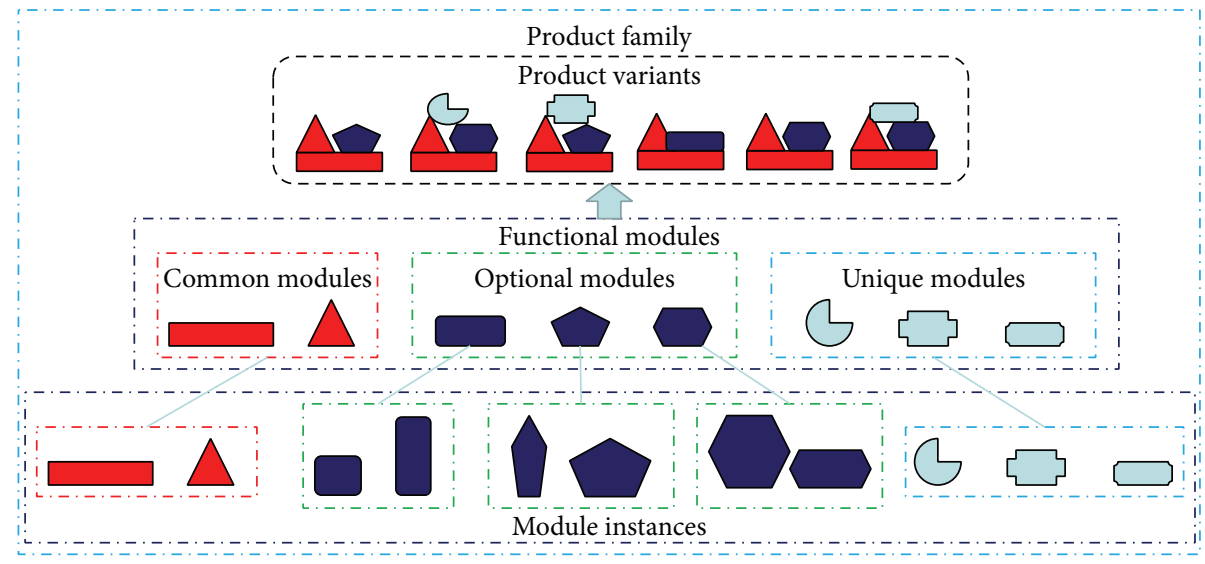

FIgURE 4: Hierarchical model of modular product family.

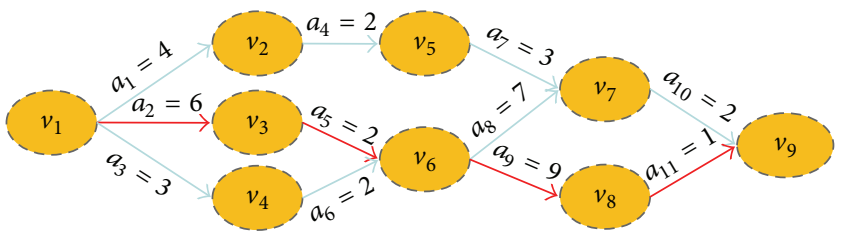

FIgURe 5: AOE-net.

module, there are several module instances with favorable interchangeability, and designers can configure different product variants to meet diversified customer requirements by specific selection and combination mechanism. As in small-sized wheel loader product family, according to the technological capacity in the cooperating company, the braking system module is shared by all the variants in the product family, so it is defined as the common module. In order to meet different requirements for power, the company purchases different power systems such as ZH4100G2, LR4A3G84X1Q, and LR4B3-G84X4Q, as shown in Figure 1, so the power system module is defined as the variant or optional module.

3.2. Critical Chain Technique. AOE-net (Activity on Edge net) represents the sequence of production activities by directed edges and is mainly utilized to estimate the completion time of the whole project and to search the critical path, by shortening the time of which the efficiency of the entire project would be improved. Therefore, the activities in PFAL could be shown as an AOE-net.

AOE-net is a weighted directed acyclic graph, where the vertices denote events, the arcs, and the corresponding weights denote activities and their duration, respectively. An AOE net including nine events $\left(v_{1}, v_{2}, \ldots, v_{9}\right)$ and eleven activities $\left(a_{1}, a_{2}, \ldots, a_{11}\right)$ is shown in Figure 5. The whole process has one and only one starting vertex $v_{1}$ and end vertex $v_{9}$; that is, the net has only one vertex with zero in-degree and only one vertex with zero out-degree. The occurrence of an event means the preceding activity has (activities have) been completed and the subsequent activity (activities) can begin.
For example, the occurrence of event $v_{6}$ means activities $a_{5}$ and $a_{6}$ have been completed and activities $a_{8}$ and $a_{9}$ can be carried out next, and the duration of activity $a_{1}$ is four units of time.

The main research issues of AOE-net include the following: (1) how much time at least is required to complete the whole project and (2) what are the key activities affecting the project progress. In an AOE-net, part of the activities can be performed simultaneously, and the shortest possible time for completing the whole project is the sum of weighted time in the longest path from the starting vertex to the end vertex, that is, the sum of duration required by the activities on the longest path which is, therefore, defined as the critical path.

Assuming the starting point is $v_{1}$, the length of the longest path from $v_{1}$ to $v_{i}$ is called the earliest starting time (EST) of the event $v_{i}$ which determines the earliest starting time of activities represented by arcs starting with vertex $v_{i}$. Here we use $e(i)$ to denote the earliest starting time (EST) and $l(i)$ to represent the latest starting time (LST) of the activity $a_{i}$, where LST means the latest time to start the activity $a_{i}$ on the premise of not delaying the whole project and the difference $(l(i)-e(i))$ between ES and LS denotes the spare time (namely, time remaining/surplus) of the activity $a_{i}$. When the time remaining/surplus equals zero, the activity $a_{i}$ has no spare time indicating it is a critical activity. Consequently, the critical activities constitute a critical path and it is important for the progress of the whole project to improve efficiency of the critical activities, while the early completion of the noncritical activities cannot speed up the progress of the project.

In Figure 5, the critical path from $v_{1}$ to $v_{9}$ is $v_{1} \rightarrow v_{3} \rightarrow$ $v_{6} \rightarrow v_{8} \rightarrow v_{9}$ with the length 18 which indicates that EST is 18 , while EST of $a_{7}$ is 6 and LST is 16 , which implies that postponing or starting the activity ahead of time by 10 units of time would not influence the progress of the whole project. Hence it is the key for improving the project progress or efficiency to analyze the critical path or activities.

The core of acquiring the critical path is to seek out the critical activity $a_{i}$ meeting the requirement of $l(i)=e(i)$. To simplify analysis, we define the earliest and latest starting time of event $v_{i}$ as $v e(i)$ and $v l(i)$, respectively. Supposing 
TABLE 1: Precedence relation between assembly tasks.

\begin{tabular}{|c|c|c|c|c|c|c|c|c|c|c|}
\hline Task & $\mathrm{A} 1$ & A2 & A3 & A4 & A5 & A6 & A7 & A8 & A9 & $\mathrm{A} 10$ \\
\hline Predecessor(s) & - & - & - & $\mathrm{A} 1, \mathrm{~A} 2, \mathrm{~A} 3$ & A4 & A5 & A6 & A6 & - & - \\
\hline Duration & 41 & 42 & 40 & 39 & 39 & 48 & 36 & 36 & 28 & 30 \\
\hline Operators assigned & 5 & 6 & 4 & 10 & 14 & 2 & 3 & 1 & 1 & 1 \\
\hline Task & A11 & $\mathrm{A} 12$ & $\mathrm{~A} 13$ & A14 & A15 & A16 & A17 & A18 & A19 & A20 \\
\hline Predecessor(s) & - & A6, A9, A10, A11 & $\mathrm{A} 12$ & A13 & $\mathrm{A} 13$ & A13 & A13 & $\mathrm{A} 14, \mathrm{~A} 15, \mathrm{~A} 16, \mathrm{~A} 17$ & A18 & A18 \\
\hline Duration & 35 & 14 & 13 & 12 & 15 & 18 & 15 & 5 & 8 & 7 \\
\hline Operators assigned & 1 & 1 & 1 & 1 & 1 & 1 & 1 & 1 & 1 & 1 \\
\hline Task & $\mathrm{A} 21$ & $\mathrm{~A} 22$ & $\mathrm{~A} 23$ & $\mathrm{~A} 24$ & $\mathrm{~A} 25$ & A26 & $\mathrm{A} 27$ & A28 & A29 & $\mathrm{A} 30$ \\
\hline Predecessor(s) & A18 & $\mathrm{A} 19, \mathrm{~A} 20, \mathrm{~A} 21$ & A22 & A18, A23 & A23 & A18 & $\mathrm{A} 25, \mathrm{~A} 26$ & A23 & A23 & A23 \\
\hline Duration & 7 & 7 & 11 & 23 & 17 & 4 & 8 & 24 & 22 & 31 \\
\hline Operators assigned & 1 & 1 & 1 & 1 & 1 & 1 & 1 & 1 & 1 & 1 \\
\hline
\end{tabular}

the activity $a_{i}$ is represented by the arc between vertex $v_{j}$ and $v_{k}$ and the time needed is $T_{j k}$, then the critical activity $a_{i}$ needs to meet the following requirements:

$$
\begin{gathered}
e(i)=v e(j), \\
l(i)=v l(k)-T_{j k} .
\end{gathered}
$$

The algorithm to acquire the critical path or activities is shown as follows:

(1) constructing and initializing an AOE-net containing $n$ vertexes;

(2) starting from the vertex $v_{1}$ and setting $v e(1)=0$ and then successively calculating EST ve(i) of all the rest vertexes by topology algorithm: if the number of vertexes in the some obtained topological sequence is less than $n$, which means a loop exists in the net, then it is impossible to acquire the critical path and the algorithm terminates, shifting to step (3), otherwise;

(3) starting from the end vertex $v_{n}$ and setting $v l(n)=$ $v e(n)$ and then successively calculating LST $v l(i)(1 \leq$ $i \leq n-1)$ of all the rest events by topology algorithm;

(4) according to $v l(i)$ and $v e(i)$ of each event (vertex), calculating $\operatorname{EST} e(i)$ and $\operatorname{LST} l(i)$ of activity $a_{i}$, if $e(i)=$ $l(i)$, then $a_{i}$ is the critical activity;

(5) determining the critical activities and path according to the results in step (4) and the algorithm terminates.

3.3. Optimization Objectives. Based on the mathematical model of product family [21], we study both product family assembly line balancing and evolution planning comprehensively. The main objective of PFAL evolution planning is to minimize change of assembly line while maximizing product assembly capability, that is, tradeoff between minimization of adjustment costs and maximization of productivity. We suppose that the existing assembly line has already balanced and needs adjustment owing to the influences of external factors such as customer requirements, product functions or quantities, materials, and technologies. The adjustment costs in evolution balancing process include costs induced by adjustment of tools, equipment, and labors, and the objective of minimizing adjustment costs $I$ is represented in the following:

$$
\begin{aligned}
I= & \alpha \Delta \operatorname{Num}_{s}+\beta \Delta \operatorname{Num}_{p}+\gamma \Delta T_{\text {total }}+\delta \Delta T_{\text {idle }} \\
& +\varepsilon \Delta T_{\text {cycle }}+\phi \Delta L+\varphi \Delta \operatorname{Num}_{w},
\end{aligned}
$$

where $\mathrm{Num}_{s}, \mathrm{Num}_{p}, T_{\text {total }}, T_{\text {idle }}, T_{\text {cycle }}, L$, and $\mathrm{Num}_{w}$, respectively, denote total number of stations, total number of assembly workers, total time of assembly, idle time, cycle time, logistical cost, and total number of tasks, while $\alpha, \beta$, $\gamma, \delta, \varepsilon, \phi, \varphi$ signify the degree of influence of each index on adjustment costs and are determined on the basis of the practical situations of enterprises and expert experiences. According to (2), the objective of minimizing adjustment costs is met only when variations of all indexes mentioned above reach their lowest.

Aiming at the optimization problem for PFAL evolution balancing, the optimization objective of evolution balancing is determined by synthesizing the four balancing objectives $Z$ proposed in [21] and the evolution objective of minimizing adjustment costs $I$, as shown in (3). Besides, the influences of product family design and critical chain techniques on assembly line evolution balancing are translated into constraint conditions and introduced into the optimization model of PFAL evolution balancing, and the evolution balancing problem is optimized by the dual-population genetic algorithm proposed in [21]:

$$
P_{\min }=\mu Z+\eta I
$$

where $\mu, \eta$ are coefficients for dimension adjustment.

3.4. Improved Dual-Population Genetic Algorithm. Product family mixed-model assembly line balancing is a typical NPhard problem with many influencing factors and complicated constraints, the number of task combination increases exponentially with the number of tasks and it is difficult to solve this type of problem using traditional optimization methods, which makes genetic algorithm one of the best choices. Therefore, in this paper we adopt the dual-population genetic algorithm proposed in literature [21] to solve the optimization 


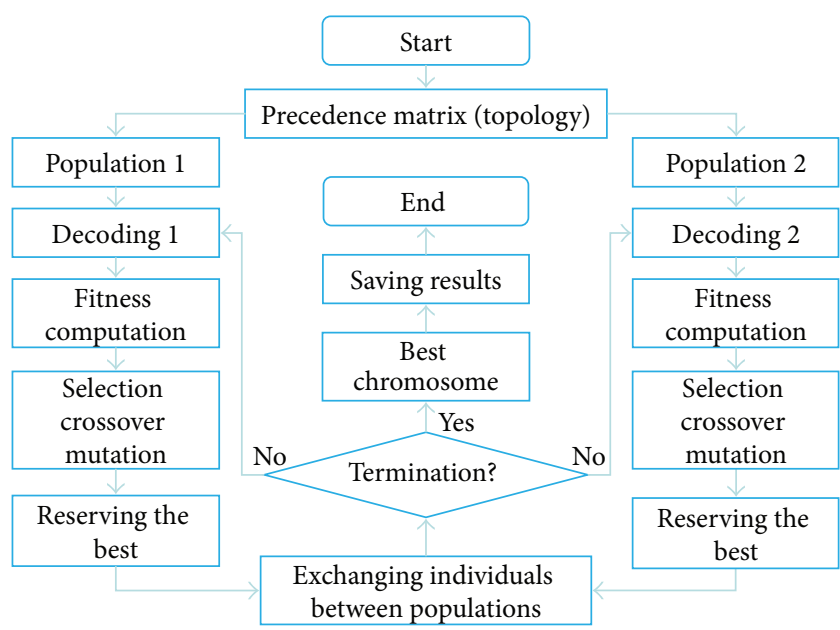

FIGURE 6: Process of dual-population genetic algorithm.

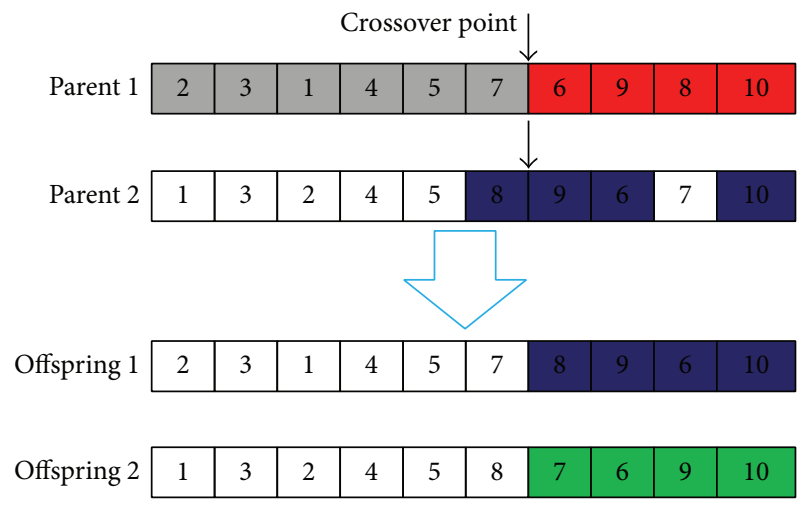

Figure 7: Process of crossover.

problem of product family assembly line. The process of this algorithm is shown in Figure 6 and the main characteristics are concluded as follows:

(1) using real number encoding method, where each gene stands for one task and the encoding length equals the total number of tasks;

(2) adopting single-point crossover mode as shown in Figure 7 and the mutation operation process as shown in Figure 8.

3.5. Evolution Balancing Optimization Process. The evolution planning process of PFAL based on product family design and critical chain techniques is shown in Figure 9 and can be implemented in the following steps.

Step 1. Based on the comprehensive precedence graph of product family, we determine that the task sequence meets the precedence order using topological sorting algorithm, convert the precedence graph into AOE-net, and then seek out the critical tasks and critical path by critical chain algorithm.

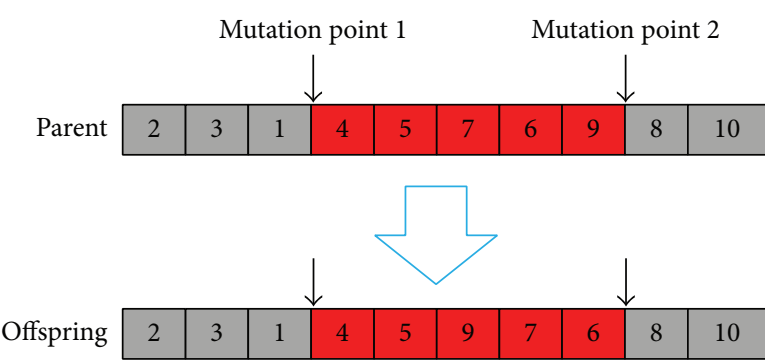

Figure 8: Process of mutation.

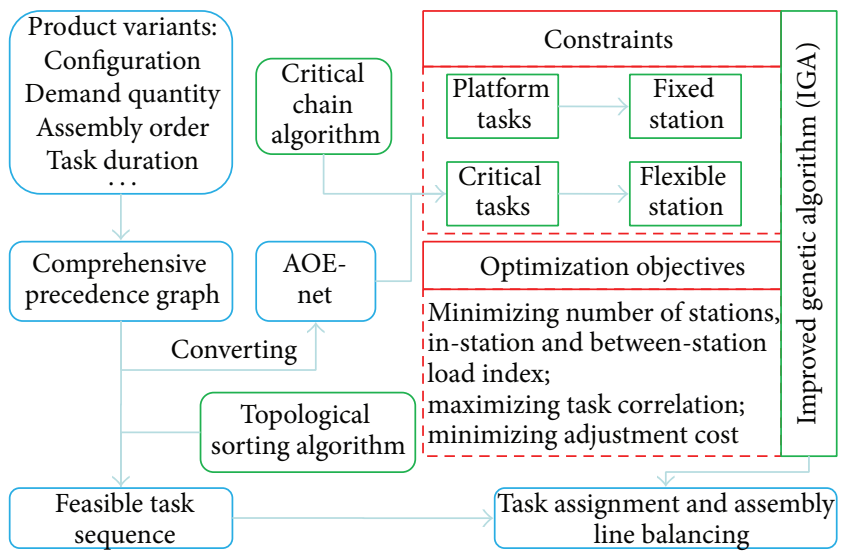

FIGURE 9: Evolution planning model of PFAL.

TABLE 2: Correlation between tasks.

\begin{tabular}{lccccc}
\hline Task & Task & Correlation & Task & Task & Correlation \\
\hline A1 & A2 & 0.8 & A15 & A18 & 0.6 \\
A1 & A3 & 0.5 & A16 & A17 & 0.7 \\
A1 & A4 & 0.1 & A17 & A18 & 0.6 \\
A1 & A5 & 0.1 & A19 & A20 & 0.5 \\
A2 & A3 & 0.7 & A19 & A21 & 0.5 \\
A2 & A4 & 0.7 & A19 & A22 & 0.5 \\
A3 & A4 & 0.8 & A19 & A23 & 0.5 \\
A4 & A5 & 0.8 & A20 & A21 & 0.5 \\
A4 & A6 & 0.4 & A21 & A22 & 0.8 \\
A5 & A6 & 0.5 & A21 & A23 & 0.5 \\
A6 & A7 & 0.1 & A22 & A23 & 0.7 \\
A6 & A12 & 0.8 & A25 & A26 & 0.7 \\
A7 & A8 & 0.5 & A25 & A27 & 0.7 \\
A9 & A12 & 0.8 & A26 & A27 & 0.4 \\
A10 & A12 & 0.8 & A28 & A29 & 0.4 \\
A11 & A12 & 0.8 & A28 & A30 & 0.4 \\
A14 & A15 & 0.7 & A29 & A30 & 0.4 \\
\hline
\end{tabular}

Step 2. We determine constraints and objectives of the evolution balancing optimization problem. Besides the constraints in the load balancing model, we add two constraints: assigning platform-related tasks to fixed stations and assigning critical tasks to flexible stations. The optimization objectives include minimizing number of stations, in-station 


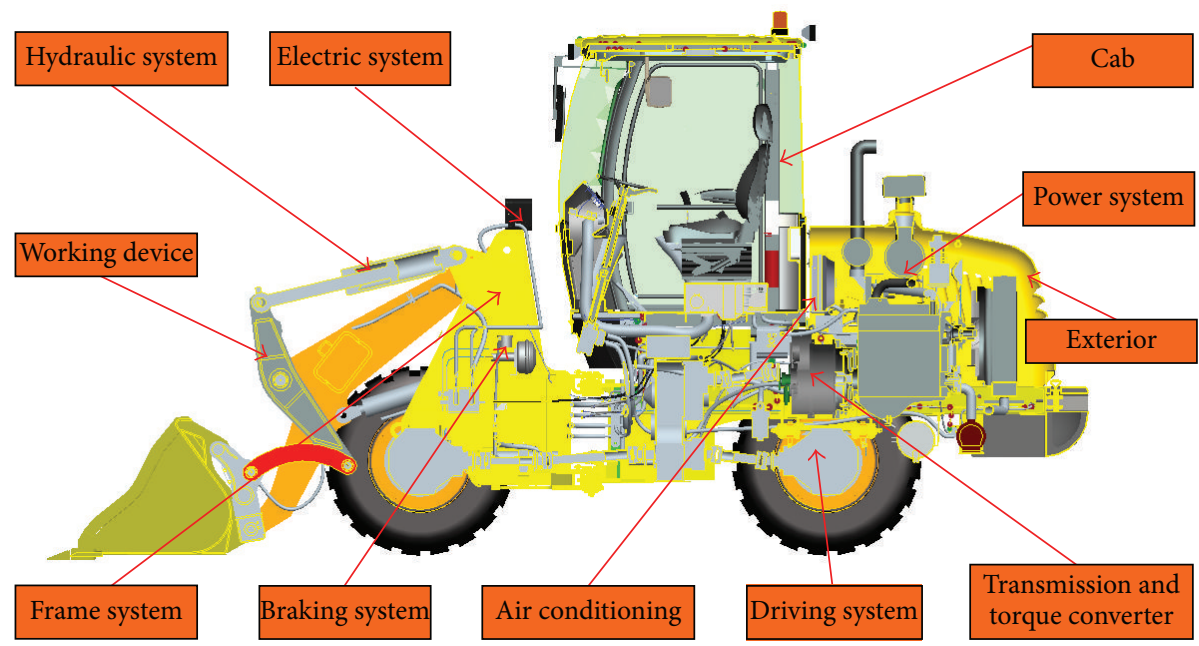

FIGURE 10: Modular architecture of small-sized wheel loader.

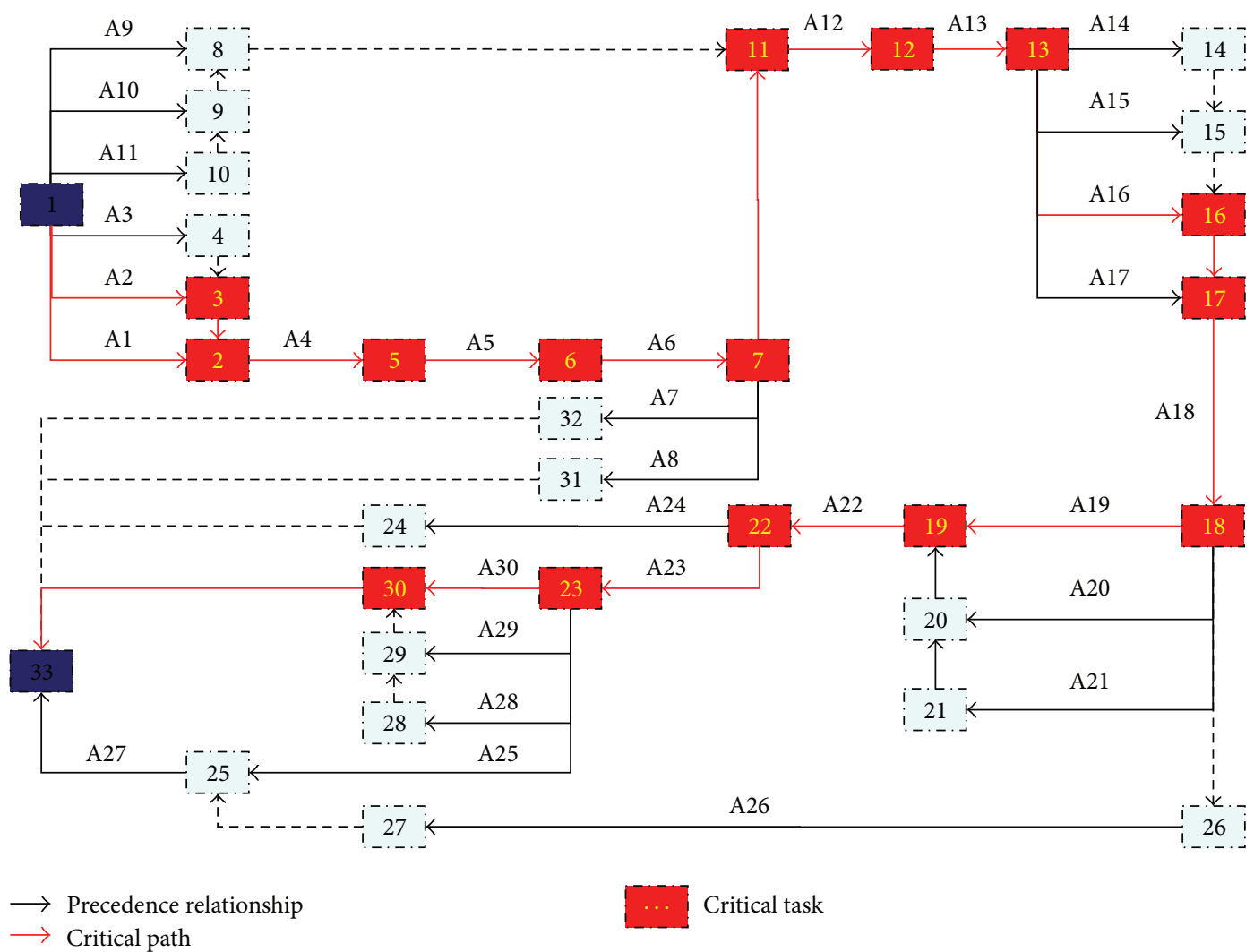

FIGURE 11: AOE-net of related tasks.

and between-station load index and adjustment cost, and maximizing task correlation.

Step 3. We assign the tasks to stations using improved dual-population genetic algorithm and obtain the optimal assignment results of PFAL evolution balancing problem.

\section{SWL Case Study}

4.1. Evolution Balancing of PFAL. In this section, the assembly line of small-sized wheel loader product family in some international enterprise [21] is restudied to verify the effectiveness and feasibility of the proposed evolution balancing model of PFAL. The small-sized wheel loader is a typical modular product family, which concludes frame system module, braking system module, driving system module, cab module, and so on, as shown in Figure 10. Different modules have one or more module instances, so the company can configure hundreds of wheel loader models to meet customer needs. Unavoidably, the functional or performance requirements of customers in different period of time changes abruptly, so the corresponding assembly line needs frequent 
TABle 3: Relevant times in AOE-net.

\begin{tabular}{lcccccccccc}
\hline Task & A1 & A2 & A3 & A4 & A5 & A6 & A7 & A8 & A9 & A10 \\
\hline LST & $\mathbf{4 1}$ & $\mathbf{4 1}$ & 80 & 80 & 119 & 167 & 274 & 274 & 167 & 167 \\
EST & $\mathbf{4 1}$ & $\mathbf{4 1}$ & 40 & 80 & 119 & 167 & 203 & 203 & 28 & 30 \\
Spare time & $\mathbf{0}$ & $\mathbf{0}$ & 40 & 0 & 0 & 0 & 71 & 71 & 139 & 137 \\
\hline Task & A11 & A12 & A13 & A14 & A15 & A16 & A17 & A18 & A19 & A20 \\
\hline LST & 167 & 181 & 194 & 212 & 212 & $\mathbf{2 1 2}$ & $\mathbf{2 1 2}$ & 217 & 225 & 225 \\
EST & 35 & 181 & 194 & 206 & 209 & $\mathbf{2 1 2}$ & $\mathbf{2 1 2}$ & 217 & 225 & 224 \\
Spare time & 132 & 0 & 0 & 6 & 3 & $\mathbf{0}$ & $\mathbf{0}$ & 0 & 0 \\
\hline Task & A21 & A22 & A23 & A24 & A25 & A26 & A27 & A28 & A29 & A30 \\
\hline LST & 225 & 232 & 243 & 274 & 266 & 260 & 274 & 274 & 274 & 274 \\
EST & 224 & 232 & 243 & 255 & 260 & 221 & 260 & 267 & 267 & 274 \\
Spare time & 1 & 0 & 0 & 19 & 6 & 39 & 14 & 7 & 7 \\
\hline
\end{tabular}

TABLE 4: Relevant tasks on the critical chain.

\begin{tabular}{lllllllllllll}
\hline Critical chain 1 & A1 & A4 & A5 & A6 & A12 & A13 & A16 & A18 & A19 & A22 & A23 & A30 \\
Critical chain 2 & A2 & A4 & A5 & A6 & A12 & A13 & A16 & A18 & A19 & A22 & A23 & A30 \\
Critical chain 3 & A1 & A4 & A5 & A6 & A12 & A13 & A17 & A18 & A19 & A22 & A23 & A30 \\
Critical chain 4 & A2 & A4 & A5 & A6 & A12 & A13 & A17 & A18 & A19 & A22 & A23 & A30 \\
\hline
\end{tabular}

TABLE 5: Relevant tasks for certain modules.

\begin{tabular}{lc}
\hline Module & Relevant tasks \\
\hline Module A & $1,2,4,5$ \\
Module B & $6,10,12$ \\
Module C & $14,15,17$ \\
Module D & $16,18,19,21$ \\
Module E & $20,22,23,26,28,29$ \\
\hline
\end{tabular}

adjustment which induces considerable cost. To lower or control the adjustment cost in product family evolution process.

The characteristics and relevant parameters of the assembly line of the small-sized wheel loader product family are described as follows.

(1) The product family contains three series of products $(916,918$, and 920T), which are assembled on the same line. The daily work time is 560 units of time and the demand quantities of 916,918 , and $920 \mathrm{~T}$ are 2,4 , and 1 , respectively; thus the cycle time CT equals 80 units of time $(=560 /(2+4+1))$.

(2) According to the precedence of assembly task, the duration of tasks, and the demand quantity of each variant, the comprehensive precedence graph can be constructed [27] and converted into the precedence relation table as shown in Table 1, including 30 tasks and the corresponding operation time (duration).

(3) When operation time of a certain task is greater than CT in the assembly process, increasing the number of operators is more conducive to making operation time less than CT.

(4) Certain correlation exists between tasks and grouping tasks with good/better correlation into the same
TABLE 6: Planning results of assembly line evolution.

\begin{tabular}{lcl}
\hline Station & Task & Balancing indexes \\
\hline 1 & 1,9 & \\
2 & 2,10 & \\
3 & 3,11 & (1) Nine stations \\
4 & 4,5 & (2) Between-station load: 21 \\
5 & $6,12,13$ & (3) In-station load: 5.1 \\
6 & $7,14,15,17$ & (4) Correlation of tasks: 4.9 \\
7 & $8,16,18,19,21$ & \\
8 & $20,22,23,26,28$ & \\
9 & $25,27,29,30$ & \\
\hline
\end{tabular}

station will bring convenience and operability to the whole assembly line. By investigation and survey in enterprise and the empirical knowledge of domain experts, correlation values between 2 of 30 tasks are shown in Table 2 while the task correlation not listed in the table all equals zero.

According to the precedence relation between tasks as shown in Table 1 and the transformation rules of AOE-net, the AOE-net of the 30 assembly tasks is constructed as shown in Figure 11.

Using the introduced algorithm of critical chain in Section 3.2, the relevant times such as EST, LST, and spare time and critical activities whose EST is equal to LST are acquired as shown in Table 3.

Four critical chains are found out and listed in Table 4, by shortening the duration of critical tasks on which would greatly improve assembly efficiency.

Via investigation in cooperative enterprise and modularity analysis, among all tasks of the 916, 918, and 920T assembly line, the tasks involved in loader platform and 
TABLE 7: Comparison of planning results.

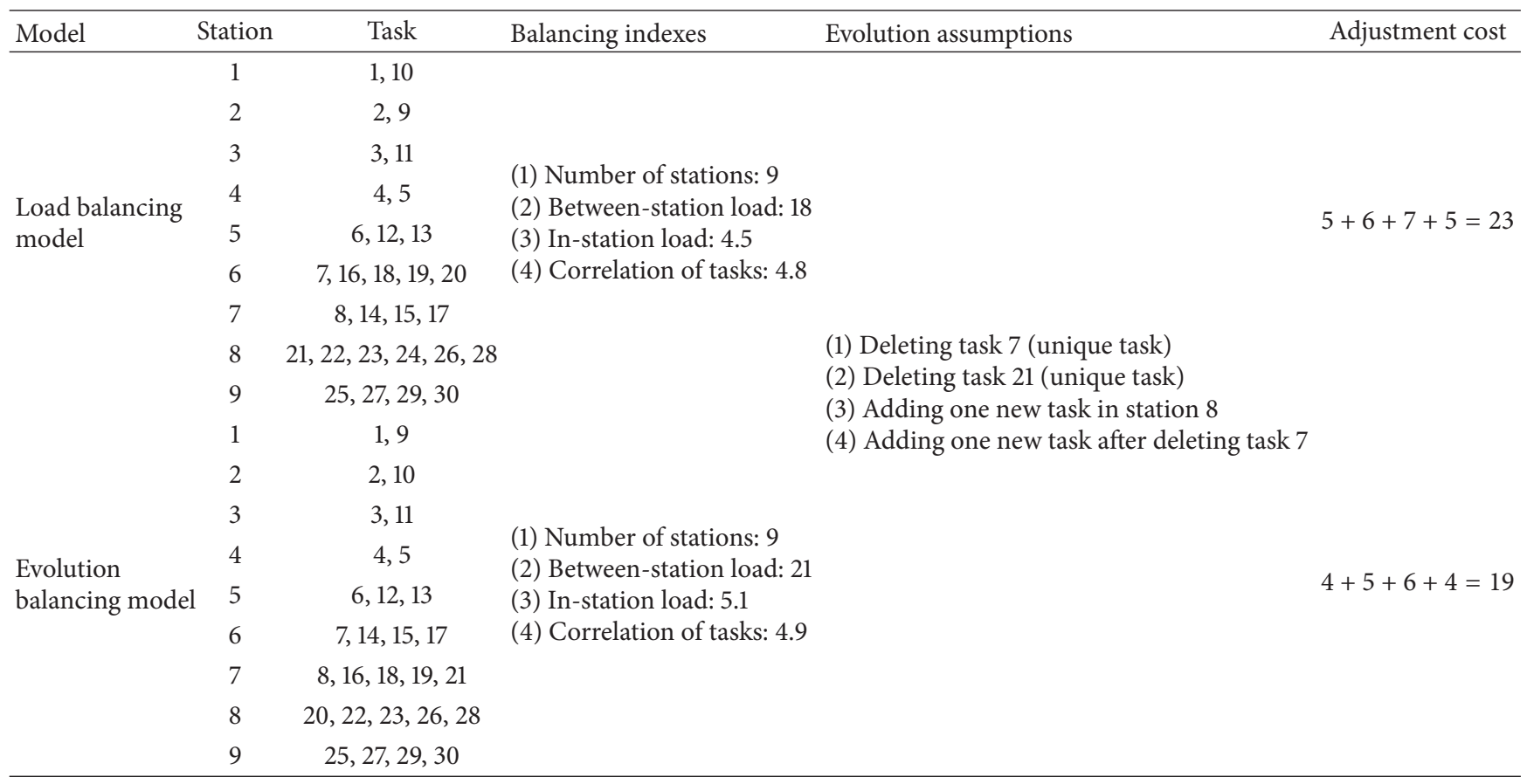

certain modules are shown in Table 5. Adding the constraints mentioned above into the improved genetic algorithm [21], the number of stations, in-station and between-station loads, and correlation between activities are optimized, meanwhile, the evolution-related objectives such as minimizing variations of quantity of operators, cycle time, and material flows are taken into consideration, and the optimization results are shown in Table 6.

4.2. Performance Analysis. To further demonstrate the effectiveness of the proposed planning model for PFAL evolution in this paper, the results are compared with those of load balancing model [21] as shown in Table 7.

It is observed from the table that, after adding constraints related to product family design and critical chain techniques, the optimized number of stations is still 9, but the betweenstation and in-station indexes have higher values, which indicates that the optimization result is not superior to that of when only considering load balancing. However, not only load balancing of PFAL but also its evolution process is evolved in this paper. Through analysis of labor cost variation induced by assembly line adjustment based on different balancing models (load balancing model or evolution balancing model), it is not difficult to find that, if the load balancing model adopted, then the adjustment cost is 23 units of labor cost, while if we use the evolution balancing model proposed in this paper, the adjustment cost is 19 units of labor cost.

Owing to individualization of customer requirement and shorter lifecycle of seasonal product family, adjustment or evolution balancing of PFAL becomes increasingly frequent; hence the benefits of evolution balancing model are much more considerable. Therefore, when dealing with assembly line balancing oriented to product family evolution process, we should not only consider loader balancing but also treat evolution planning as one more important optimization objective.

\section{Conclusions}

(1) Based on discussion of product family evolution process and its influencing factors, the dynamic evolution process of PFAL (coevolution of product family and its assembly line) is analyzed and illustrated by the case of SWL product family; combined with characteristics of PFAL and its balancing research status, the evolution balancing problem of PFAL is focused on in this paper.

(2) Product platform/key functional modules (such as the working device module or platform) change with a very small probability in product family evolution process and it is the key to raise efficiency of critical tasks (such as assembling accessory module instances) for improvement of the overall assembly efficiency; therefore, influences of the product family design techniques and critical chain technology on task assignment and assembly line balancing are discussed comprehensively and translated into constraint conditions in the evolution balancing model of PFAL.

(3) The evolution balancing problem of PFAL is optimized using an intelligent evolutionary algorithm (dual-population GA) and, compared with the traditional load balancing model, results of the SWL case show that, in the constant evolution process of product family, adjustment costs of PFAL reduce significantly using the evolution balancing model with other indicators largely unchanged, which generates considerable economic benefits for enterprises. 
(4) The research on evolution balancing focuses on the issue of assembly line adjustment when the current assembly line has already balanced but was disturbed by external dynamic factors with more planning constraints and much more difficulty. Therefore, how to fully consider all factors such as tradeoff between load balancing indicators and adjustment costs, analysis of other adjustment costs besides labor cost, and so on and then to establish an objective model more precisely for the PFAL evolution balancing is an interesting perspective, in which further research is needed.

\section{Conflict of Interests}

The authors declare that there is no conflict of interests regarding the publication of this paper.

\section{Acknowledgments}

This work was financially supported by the National Natural Science Foundation of China (71172055) and the Special Program for Innovation method of the Ministry of Science and Technology, China (Grant no. 2011IM020400).

\section{References}

[1] M. Azoulay, A. Veprik, V. Babitsky, and N. Halliwell, "Distributed absorber for noise and vibration control," Shock and Vibration, vol. 18, no. 1-2, pp. 181-219, 2011.

[2] Y. Lei, J. Lin, and Z. He, "Fault diagnosis of planetary gearboxes based on multi-sensor information fusion," Journal of Vibration and Shock, vol. 29, pp. 216-218, 2010.

[3] J. Xiang, X. Cui, Y. Wang et al., "Optimized stochastic resonance method for bearing fault diagnosis," Transactions of the Chinese Society of Agricultural Engineering, vol. 30, pp. 50-55, 2014.

[4] Y. Lei, T. Wei, D. Kong et al., "Vibration signal simulation and fault diagnosis of planetary gearboxes based on transmission mechanism analysis," Chinese Journal of Mechanical Engineering, vol. 50, pp. 61-68, 2014.

[5] W. Zhou and D. Li, "Improving performance of cantilevered momentum wheel assemblies by soft suspension support," Shock and Vibration, vol. 20, no. 4, pp. 737-748, 2013.

[6] J. Lu, Y. Xu, C. Hu, A. F. Vakakis, and L. A. Bergman, "5DOF dynamic model of vehicle shimmy system with clearance at universal joint in steering handling mechanism," Shock and Vibration, vol. 20, no. 5, pp. 951-961, 2013.

[7] J. Xiang, Z. Jiang, and X. Chen, "A class of wavelet-based Rayleigh-Euler beam element for analyzing rotating shafts," Shock and Vibration, vol. 18, no. 3, pp. 447-458, 2011.

[8] M. E. Salveson, “The assembly line balancing problem," Journal of Industrial Engineering, vol. 6, pp. 18-25, 1955.

[9] J. Bukchin, E. M. Dar-El, and J. Rubinovitz, "Mixed model assembly line design in a make-to-order environment," Computers \& Industrial Engineering, vol. 41, no. 4, pp. 405-421, 2001.

[10] E. Erel, I. Sabuncuoglu, and H. Sekerci, "Stochastic assembly line balancing using beam search," International Journal of Production Research, vol. 43, no. 7, pp. 1411-1426, 2005.

[11] A. Scholl and C. Becker, "State-of-the-art exact and heuristic solution procedures for simple assembly line balancing," European Journal of Operational Research, vol. 168, no. 3, pp. 666693, 2006.
[12] C. Merengo, F. Nava, and A. Pozzetti, "Balancing and sequencing manual mixed-model assembly lines," International Journal of Production Research, vol. 37, no. 12, pp. 2835-2860, 1999.

[13] A. N. Haq, K. Rengarajan, and J. Jayaprakash, "A hybrid genetic algorithm approach to mixed-model assembly line balancing," The International Journal of Advanced Manufacturing Technology, vol. 28, no. 3-4, pp. 337-341, 2006.

[14] Y. Bukchin and I. Rabinowitch, "A branch-and-bound based solution approach for the mixed-model assembly line-balancing problem for minimizing stations and task duplication costs," European Journal of Operational Research, vol. 174, no. 1, pp. 492-508, 2006.

[15] U. Özcan and B. Toklu, "Balancing of mixed-model two-sided assembly lines," Computers and Industrial Engineering, vol. 57, no. 1, pp. 217-227, 2009.

[16] S. Akpınar and G. M. Bayhan, "A hybrid genetic algorithm for mixed model assembly line balancing problem with parallel workstations and zoning constraints," Engineering Applications of Artificial Intelligence, vol. 24, no. 3, pp. 449-457, 2011.

[17] S. Akpinar, G. M. Bayhan, and A. Baykasoglu, "Hybridizing ant colony optimization via genetic algorithm for mixed-model assembly line balancing problem with sequence dependent setup times between tasks," Applied Soft Computing Journal, vol. 13, no. 1, pp. 574-589, 2013.

[18] W. Xu and T. Xiao, "Strategic robust mixed model assembly line balancing based on scenario planning," Tsinghua Science and Technology, vol. 16, no. 3, pp. 308-314, 2011.

[19] B. Yagmahan, "Mixed-model assembly line balancing using a multi-objective ant colony optimization approach," Expert Systems with Applications, vol. 38, no. 10, pp. 12453-12461, 2011.

[20] N. Manavizadeh, M. Rabbani, D. Moshtaghi, and F. Jolai, "Mixed-model assembly line balancing in the make-to-order and stochastic environment using multi-objective evolutionary algorithms," Expert Systems with Applications, vol. 39, no. 15, pp. 12026-12031, 2012.

[21] L. Hou, Y.-M. Wu, R.-S. Lai, and C.-T. Tsai, "Product family assembly line balancing based on an improved genetic algorithm," The International Journal of Advanced Manufacturing Technology, vol. 70, no. 9-12, pp. 1775-1786, 2014.

[22] L. Hou, Y.-M. Wu, R.-S. Lai, and S. H. Huang, "Evaluation and driver analysis in product family evolution," The International Journal of Advanced Manufacturing Technology, vol. 69, no. 1-4, pp. 863-874, 2013.

[23] F. Kimura and J. Nielsen, "A design method for product family under manufacturing resource constraints," CIRP AnnalsManufacturing Technology, vol. 54, no. 1, pp. 139-142, 2005.

[24] A. Bryan, J. Ko, S. J. Hu, and Y. Koren, "Co-evolution of product families and assembly systems," CIRP Annals-Manufacturing Technology, vol. 56, no. 1, pp. 41-44, 2007.

[25] L. Hou, R. Tang, and Y. Xu, "Review of theory, key technologies and its application of modular product design," Chinese Journal of Mechanical Engineering, vol. 40, no. 1, pp. 56-61, 2004.

[26] M. H. Meyer and A. P. Lehnerd, The Power of Product PlatformBuilding Value and Cost Leadership, The Free Press, New York, NY, USA, 1997.

[27] Z. Zhang, W. Cheng, B. Zhong, and J. Wang, "Hybrid behavior ant colony optimization for mixed-model assembly line balancing problem," Journal of Mechanical Engineering, vol. 45, no. 5, pp. 95-101, 2009. 

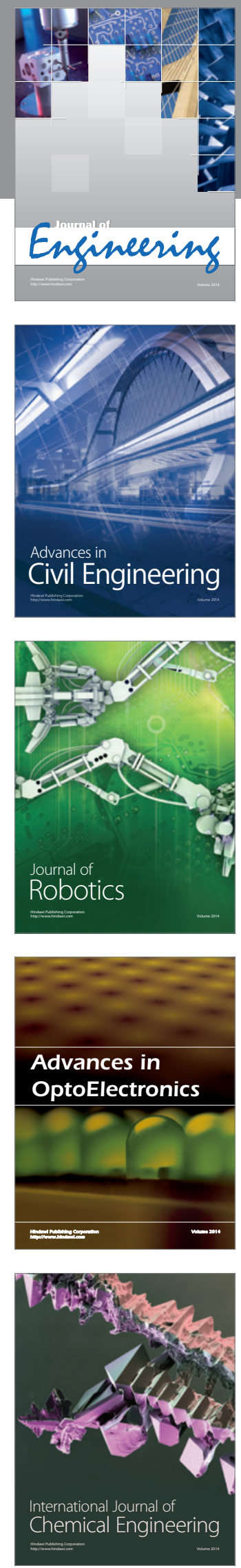

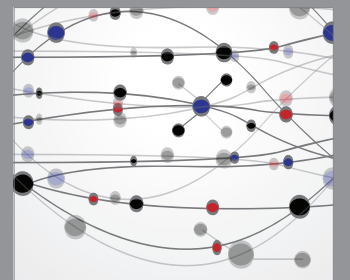

The Scientific World Journal
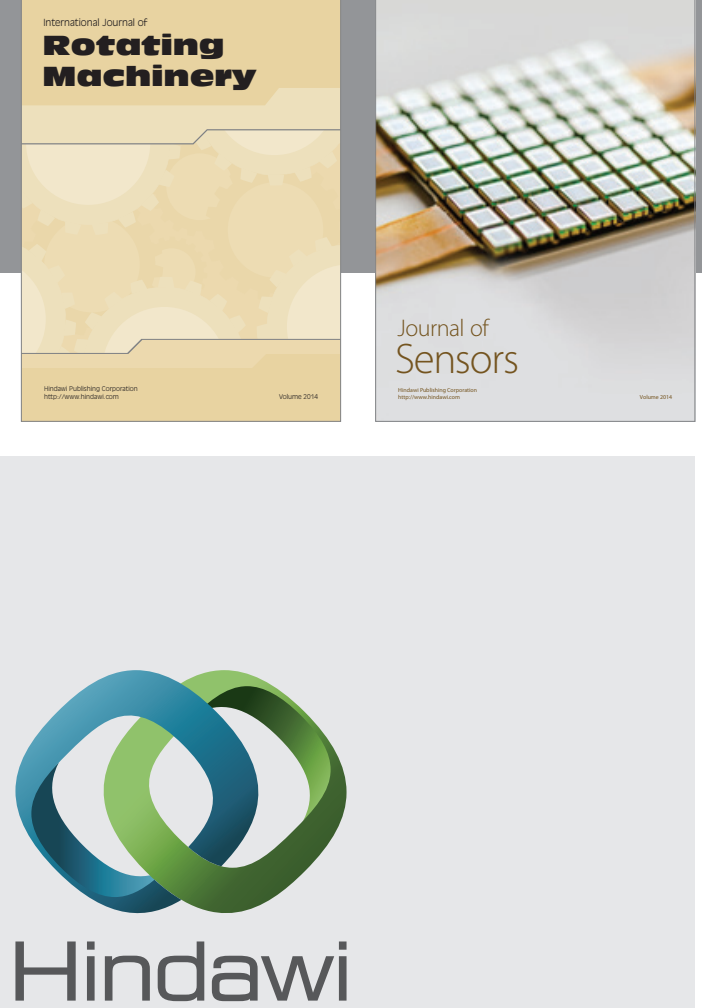

Submit your manuscripts at http://www.hindawi.com
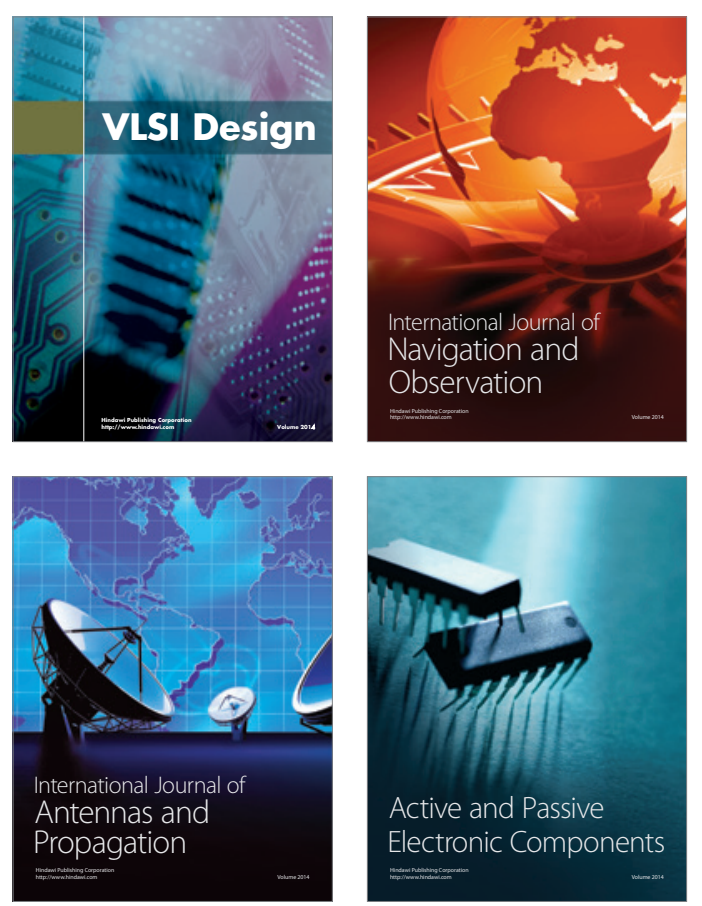
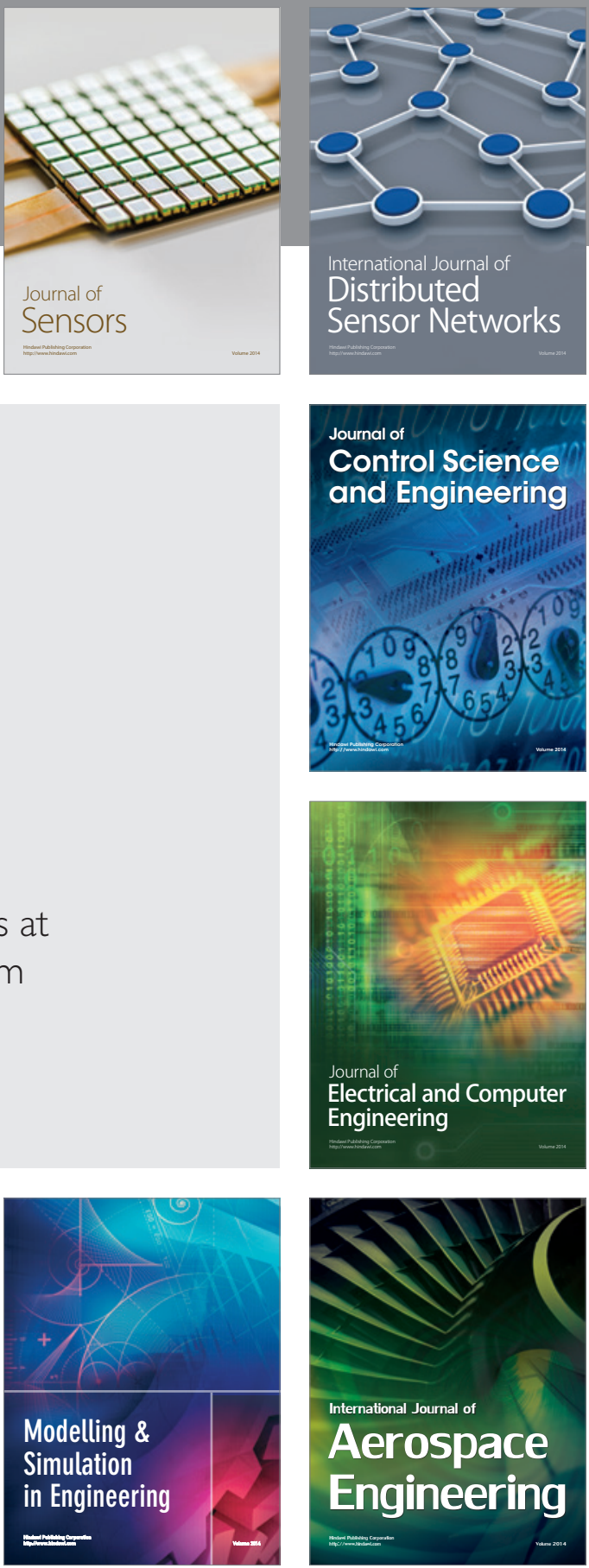

Journal of

Control Science

and Engineering
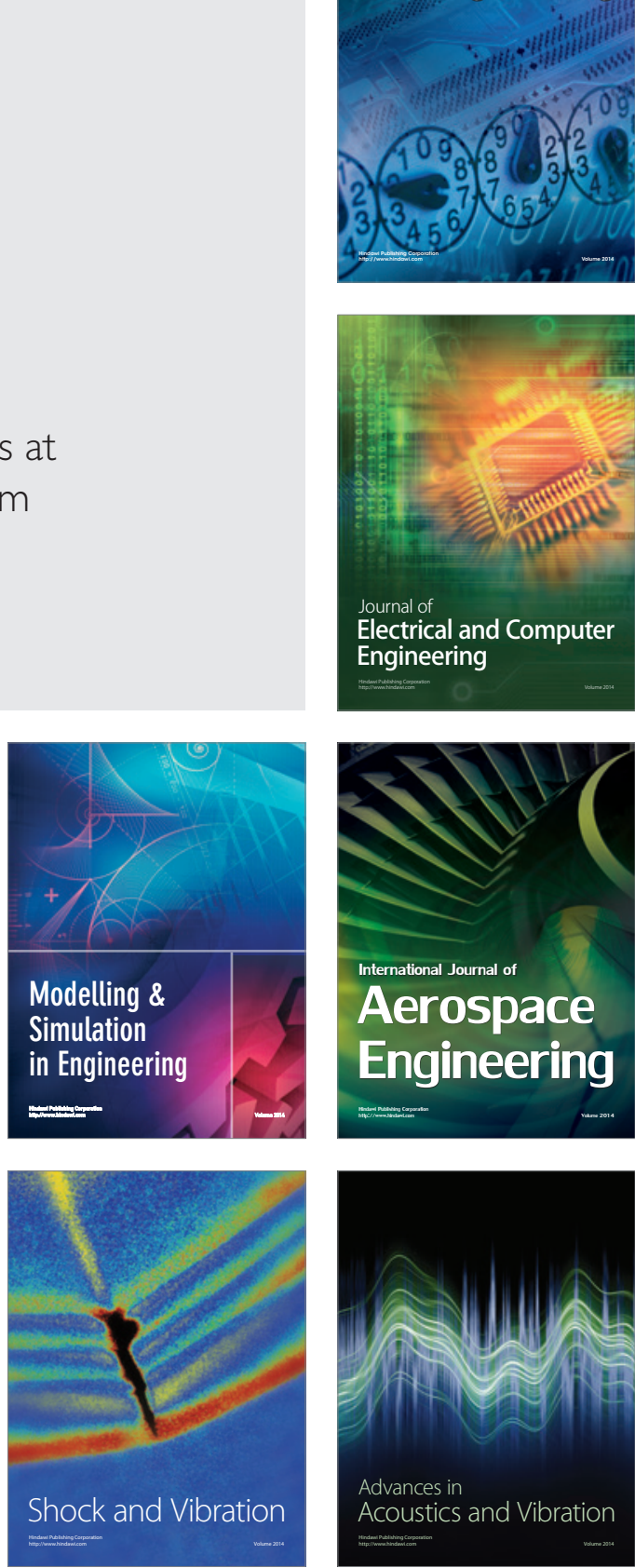